\title{
Models of current sintering
}

\author{
Sebastian Angst ${ }^{1, \star}$, Lukas Engelke ${ }^{1}$, Markus Winterer ${ }^{2}$, and Dietrich E. Wolf ${ }^{1, \star \star}$ \\ ${ }^{1}$ Faculty of Physics and CENIDE, University of Duisburg-Essen, D-47057, Duisburg, Germany \\ ${ }^{2}$ Nanoparticle Process Technology and CENIDE, University of Duisburg-Essen, D-47057, Duisburg, Germany
}

\begin{abstract}
Densification of (semi-)conducting particle agglomerates with the help of an electrical current is much faster and more energy efficient than traditional thermal sintering or powder compression. Therefore, this method becomes more and more common among experimentalists, engineers, and in industry. The mechanisms at work at the particle scale are highly complex because of the mutual feedback between current and pore structure. This paper extends previous modelling approaches in order to study mixtures of particles of two different materials. In addition to the delivery of Joule heat throughout the sample, especially in current bottlenecks, thermoelectric effects must be taken into account. They lead to segregation or spatial correlations in the particle arrangement. Various model extensions are possible and will be discussed.
\end{abstract}

\section{Introduction}

Current-activated pressure-assisted densification (CAPAD) is used to rapidly densify (semi-)conducting powders with little grain growth, which enables an upscalable production of nanostructured bulk material [1,2]. A low pressure in the range of $10-100 \mathrm{MPa}$ is applied, while a kA current is flowing through the initially porous powder. The pressure alone is too weak for a significant densification. Dissipation of electrical energy is used to heat up the powder, which enables fast and almost complete densification.

The mechanisms underlying current "sintering" are very controversial. Therefore the experimental procedure is known under several different names: Field assisted sintering technology (FAST), spark plasma sintering (SPS), and current-activated pressure-assisted densification (CAPAD) [3], which we adopt in this paper. Especially during early stages of the process particle rearrangement is among the relevant densification mechanisms $[2,4,5]$.

Several approaches to simulate CAPAD were developed in recent years, among them thermo-electricalmechanical finite element studies [6, 7]. Furthermore, network models have been investigated, which focus on the complex feedback between microstructure, current flow and densification $[8,9]$, but do not address issues of ordinary thermal sintering such as solid neck growth at particle contacts, grain coarsening and pore shrinkage by grain boundary diffusion [10], which may also play a role in CAPAD.

Here, we report recent results obtained with an extension of this network approach to binary particle mixtures. We predict that the Peltier effect leads to correlated particle rearrangement. If the porosity is high enough, this may

\footnotetext{
^e-mail: sebastian.angst@uni-due.de
}

$\star \star$ e-mail: dietrich.wolf@uni-due.de result in segregation of the two components. As an outlook we expand the view even further and discuss recent experiments, where phases of different conductivity are formed via current driven segregation and second phase formation during the densification process. Finally, we sketch, how we include heat transport through pores of the particle agglomerate.

\section{Model}

The model is based on the Onsager-de Groot-Callen theory of thermoelectricity (e.g. [11]), which provides a description of the electrical current density $\mathbf{j}$ and the heat current density $\mathbf{j}_{\mathbf{Q}}$ :

$$
\begin{aligned}
\mathbf{j} & =-\sigma(\nabla \mu / q+\alpha \nabla T) \\
\mathbf{j}_{\mathbf{Q}} & =\pi \mathbf{j}-\kappa \nabla T .
\end{aligned}
$$

$\sigma$ being the electrical conductivity, the first term on the right hand side of eq. (1) is Ohm's law, describing electrical current due to a gradient of the electrochemical potential $\mu$. According to the Seebeck effect, a temperature gradient $\nabla T$ drives mobile charge carriers as well, which is expressed by the second term with the Seebeck coefficient $\alpha$. The second term in eq. (2) is Fourier's law of heat conduction with the heat conductivity $\kappa$. The first term describes the Peltier effect that electrical currents transport also heat proportional to the Peltier coefficient $\pi$, which is related to the Seebeck coefficient via the Kelvin relation $\pi=\alpha T$. The time evolution of the local temperatures $T$ follows from

$$
c \frac{\partial T}{\partial t}+\nabla \cdot \mathbf{j}_{\mathrm{Q}}=-\frac{\nabla \mu}{q} \cdot \mathbf{j},
$$

where $c$ denotes the specific heat capacity times mass density. The right hand side is the electrical power density, 


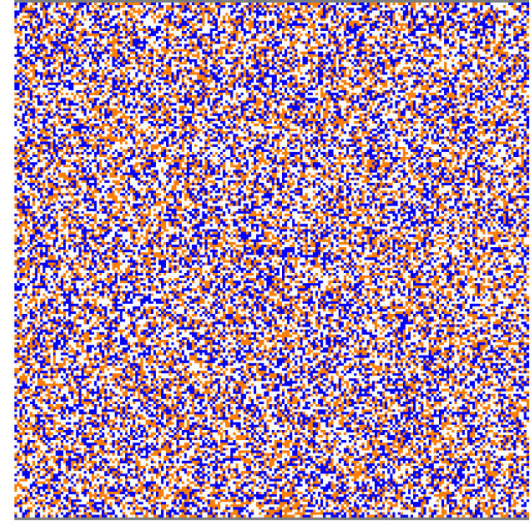

(a) $t=0$

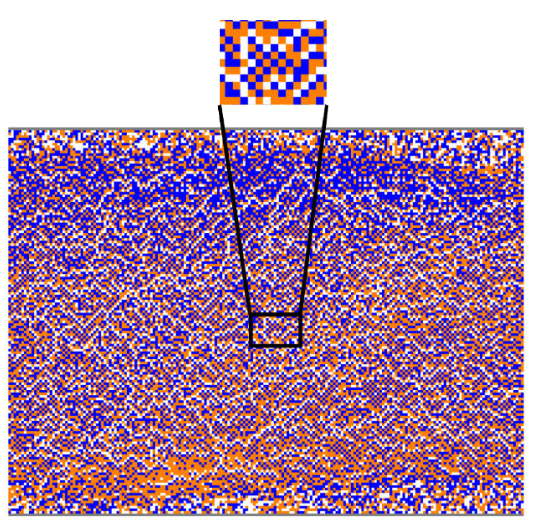

(b) $t=500$

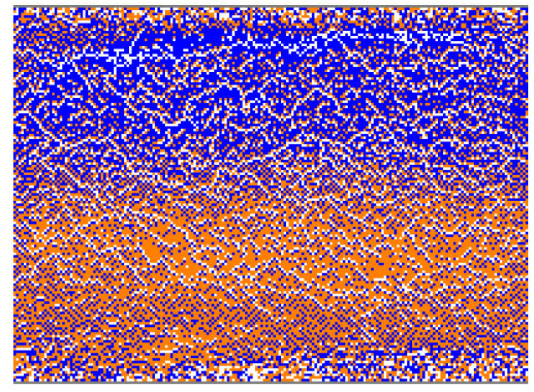

(c) $t=2000$

Figure 1: Configurations of a CAPAD simulation with two different particle types, type $\mathrm{B}$ (blue) and type $\mathrm{O}$ (orange). We choose $\alpha_{\mathrm{B}}=1$ and $\alpha_{\mathrm{O}}=0$, while all other transport parameters are equal. The number of particles of type $\mathrm{B}$ and of type $\mathrm{O}$ are equal. The current $I$ is flowing from the bottom to the top. Time is measured in units of the heat capacity $C$ divided by the heat conductance $K$, which are the same for all particles in this simulation.

which contributes to the temperature evolution as a source term. Compared to heat diffusion, charges redistribute extremely fast. Therefore it is reasonable to assume that the local electrochemical potentials $\mu$ adapt instantaneously to the temperature change. They are obtained from Kirchhoff's first law,

$$
\nabla \cdot \mathbf{j}=0
$$

In this paper we present only two-dimensional simulations in order to explain the ideas most clearly. We use the network model defined more generally in [12] to discretize the above equations on a square lattice. Two particle types are randomly distributed on the lattice sites with an initial solid volume fraction $\rho=0.6$. Both particle types occur with the same probability. At the top and the bottom, electrodes are located and kept at a constant heat bath temperature $T_{\mathrm{HB}}$.

A particle $i$ is characterized by the electrochemical potential $\mu_{i}$, temperature $T_{i}$ and heat capacity $C_{i}$, while the bonds carry the transport coefficients. Solving the discretized versions of eqs. (3) and (4), we obtain the time evolution of $\mu_{i}$ and $T_{i}$. If a particle's temperature exceeds a mobilization threshold $T_{\mathrm{m}}$, reduced friction allows it to move into a neighboring void, i.e. to a randomly chosen empty neighbor site [8]. If several particles are mobilized at the same time, they move in random order. As a result of the particle motion the connection between the electrodes may be lost. In this case the upper electrode and all particles connected to it move downwards until percolation is reestablished.

It is assumed that the particle reorganization, once the reduced friction renders the system mechanically unstable, happens very fast compared to heat diffusion. Therefore in the present model the particle displacement to a neiboring empty site takes just one time step. A more accurate representation of the particle dynamics by discrete element methods was tested [9], but would be too time consuming for the exploratory study intended here.
In this work, the two different types of particles have identical properties except the Seebeck coefficient. As natural units we use the heat bath temperature $T_{\mathrm{HB}}$, the electrical conductance of a bond $G$, the heat conductance $K$, the heat capacity of a site $C$ and the particle diameter. We set $T_{\mathrm{m}}=1.1$ and find that $I=1$ is a reasonable value for the electrical current, which is flowing from bottom to top electrode.

\section{Segregation in binary mixtures}

\subsection{Time evolution of the structure}

Figure 1 shows snapshots of a binary mixture on a $200 \times$ 200 square lattice. B-particles marked blue have $\alpha_{\mathrm{B}}=1$ and O-particles marked orange are characterized by $\alpha_{\mathrm{O}}=$ 0 . While in the initial configuration particles are homogeneously distributed, we recognize a slight segregation at $t=500$ (see fig. 1b). In the mixed central region, strong correlations between B- and O-particles emerge. Some areas exhibit a checkerboard-like pattern (CBP), which is a lattice artifact, but indicates the spatial correlations that are expected to emerge also without a lattice. At $t=2000$ the strength of segregation has increased even further and is clearly visible (see fig. 1c). Particles with the higher Peltier coefficient (blue) are concentrated above, those with low Peltier coefficient (orange) below. As the current flows from the bottom to the top, it follows that it carries less heat towards the center region than away from it. This explains that Peltier cooling occurs in between the segregated areas. We checked that these patterns are caused by the Peltier effect (see below).

The mechanism for the pattern formation becomes clear, when one considers the mobility of a local configuration, which requires that the mobilization temperature is exceeded and empty neighboring sites are available. Due to the Peltier effect, O-B-interfaces in current direction are cooled, while B-O-interfaces are heated. B-B- or O-Ointerfaces do not experience any interface Peltier effect. 


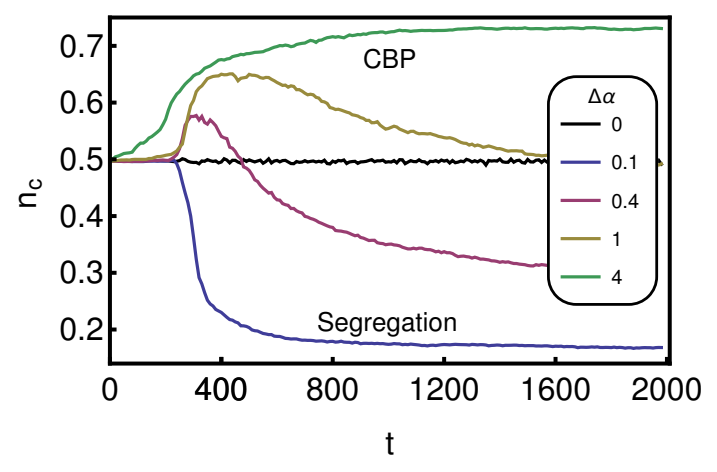

Figure 2: The relative amount of contacts between different types of particles over time. For large $\alpha_{\mathrm{B}}$ the CBP occurs rapidly and is maintained for the whole simulation time, while for intermediate $\alpha_{\mathrm{B}}$ the CBP vanishes with time. For small $\alpha_{\mathrm{B}}$ particles segregate rapidly, while for large $\alpha_{\mathrm{B}}$ the process is much slower.

As a consequence, it is more likely that particles having a B-O-interface become mobile, and their elimination leads to more stable configurations. However, in fig. 1c one still finds regions with $\mathrm{CBP}$, which suggests that the $\mathrm{CBP}$ is stable as well. Considering a B-particle surrounded by O-particles (or vice versa), and assuming isothermal conditions, we deduce that due to current conservation the Peltier heating and cooling at the four interfaces neutralize each other. In general however, temperatures in neighboring particles differ, leading to a net cooling or heating of the center particle in a CBP. As we will show below, cooled configurations are more likely. Starting from the initial, random configuration, the creation of a CBP gets rid of Peltier heating.

\subsection{Influence of the Seebeck coefficient difference}

Now, we discuss the influence of the Seebeck coefficient difference $\Delta \alpha=\alpha_{\mathrm{B}}-\alpha_{\mathrm{O}}=\alpha_{\mathrm{B}}$ and show that depending on $\Delta \alpha$ the self-organization leads to different configurations. Therefore we consider the amount of contacts between particles of different types $C_{\mathrm{CB}}$ divided by the total number of contacts $C$ (see fig. 2), $n_{\mathrm{c}}=C_{\mathrm{CB}} / C$. In

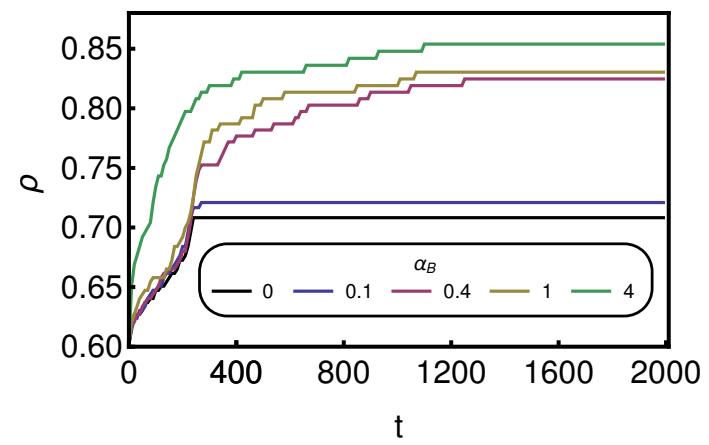

Figure 3: Solid volume fraction $\rho$ in dependence of time for various $\alpha_{\mathrm{B}}$. a perfect checkerboard pattern it reaches 1 , while it approaches 0 , if the distinct particle types arrange in two separate stripes. We verified that the stripes are oriented as indicated in fig. 1. From fig. 2 we recognize that in the initial configurations approximately half of the contacts are between equal and the other half between distinct particles. If both particles have the same Seebeck coefficient, here $\alpha_{\mathrm{O}}=\alpha_{\mathrm{B}}=0$, none of the two patterns are created, and $n_{\mathrm{c}}$ fluctuates around 0.5. For $\Delta \alpha \neq 0, n_{\mathrm{c}}$ changes significantly at $t \approx 220$ for all $\alpha_{\mathrm{B}}$ except $\alpha_{\mathrm{B}}=4$, where strong Peltier heating leads to a faster increase. Considering $\alpha_{\mathrm{B}}<4, n_{\mathrm{c}}$ grows at first, but decreases after reaching a maximum, which agrees well with the observations from the snapshots in fig. $1\left(\alpha_{\mathrm{B}}=1\right)$. In contrast, for $\alpha_{\mathrm{B}}=4$ the $\mathrm{CBP}$ is preserved.

In order to understand the pattern evolution, it is instructive to consider the time dependence of solid volume fraction $\rho$ (see fig. 3) and the amount of mobile particles $n_{\mathrm{m}}$ (see fig. 4). In the beginning, two different behaviors are present: one strongly affected by Joule $\left(\alpha_{\mathrm{B}} \lesssim 1\right)$ and the other by Peltier heating $\left(\alpha_{\mathrm{B}} \gtrsim 1\right)$. For small Seebeck coefficients, $\rho$ and $n_{\mathrm{m}}$ behave similar to $\alpha_{\mathrm{B}}=0$. Although Joule heating dominates, the Peltier effect does have an impact on the pattern formation (see fig. 2) Joule heating leads to a large amount of mobile particles. Due to their random motion, it is improbable that the percolation is interrupted so that densification is inhibited. Segregation, on the other hand, is then promoted by the prevailing low density.

Considering large $\alpha_{\mathrm{B}}=4$, Peltier-driven compaction is observed. Particles are mobilized rapidly due to strong Peltier heating, leading to a strong and fast densification at the beginning of the process. Peltier heated connections are unstable and vanish, but Peltier cooled contacts remain withdrawing heat from the sample, which keeps the total amount of mobile particles relatively small.

In order to underline the role of Peltier cooling, we examine the mean heat per particle at $t=2000$ distinguishing between average Peltier heat $\bar{P}_{\mathrm{P}}$ and average Joule heat $\bar{P}_{\mathrm{J}}$ (see inset of fig. 4). The configurations have evolved such that the Peltier heat averaged over all particles is negative,

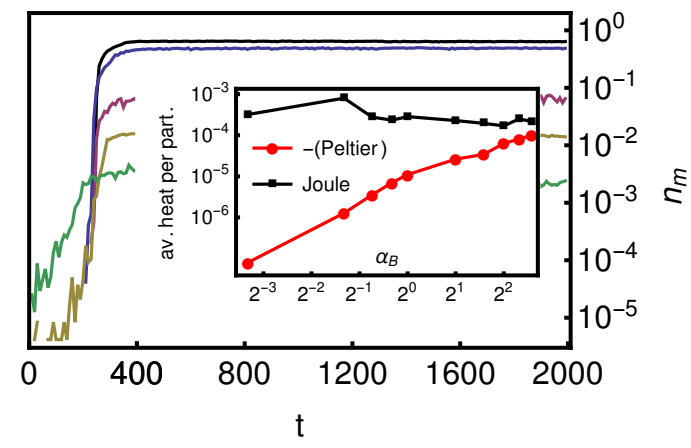

Figure 4: Relative amount of mobile particles $n_{\mathrm{m}}$ in dependence of time for various $\alpha_{\mathrm{B}}$. The inset shows the mean heat per particle distinguishing between Peltier and Joule heat. Color-coding of $\alpha_{\mathrm{B}}$ as in fig. 3 . 
$\bar{P}_{\mathrm{P}}<0$. For large $\alpha_{\mathrm{B}}, \bar{P}_{\mathrm{J}}$ and $-\bar{P}_{\mathrm{P}}$ are of the same order of magnitude, which explains the low $n_{\mathrm{m}}$. In combination with the high density, this prohibits further restructuring and CBP becomes a stationary pattern.

\section{Conclusion and Outlook}

By means of simulations we investigated current-activated pressure-assisted densification of binary particle mixtures. We discovered pattern formation due to a difference in the Peltier coefficients of both materials. Within our model, the Peltier heat created at particle interfaces determines the stability of a local configuration. If the Peltier effect predominantly cools a particle, its position is more likely to be stable and vice versa. This basic effect together with the total amount of mobile particles and the available void space determines, whether the random mixture of the two species becomes more uniform (here: a checkerboardpattern) or segregates into separate stripes.

In conclusion, we observed pattern formation in current sintering, when a local property is switched by the electrical current and is influencing the sintering process. Therefore, we predict a large effect especially for systems where the local property is directly coupled to the electrical current, such as electrical conductivity. This should be the case, when highly doped $\mathrm{Al}: \mathrm{ZnO}$ is treated by CAPAD [13]. In this case, the creation of a secondary phase is crucial to the process as will be explained in the following.

In bulk $\mathrm{ZnO}, \mathrm{Al}$ is virtually insoluble up to high temperatures. However, this does not necessarily apply to $\mathrm{ZnO}$ nanoparticles where the solubility might be much higher. Alternatively, the high dopant concentration in the $\mathrm{ZnO}$ nanoparticles could be frozen in due to their synthesis. In any case, there is experimental evidence that the sintering process destabilizes the solid solution locally and leads to formation of a separate spinel phase. This has a twofold impact on the electrical transport. On the one hand, spinel is an insulator, and on the other hand, the $\mathrm{Al}$ concentration in the vicinity of the spinel phase is decreased reducing the electrical conductivity of the doped nanoparticles.

We already know that in the early stages of current sintering spatial variations of the electrical conductivity and the microstructure can lead to the occurrence of hot spots [8]. We expect in this case that the increased temperature facilitates spinel formation which in turn reduces the electrical conductivity of the cross section in which the spinel is located. Hence, we postulate that Joule heating of this cross section increases which causes the spinel to grow in bands perpendicular to the general direction of electrical current, as observed. If, furthermore, the spinel phase limits the mobility of the particles and grain growth, this would explain the observation that the spinel-rich bands retain a porous, particle-like structure. Once spinel has formed, $\mathrm{Al}$ ions diffuse towards the precipitate and get bound there, which depletes the $\mathrm{Al}$ concentration in vicinity of spinel.
For the purpose of checking these hypotheses, several extensions of the model discussed in this paper are needed: The Onsager network must not only deal with electrical and heat transport, but include also the transport of $\mathrm{Al}$ atoms. The coarsening of the particles must be implemented in a way that is not restricted to stress-free tree-like agglomerates [14]. The displacement dynamics of the particles should be simulated with discrete element methods as in [9].

Last but not least, heat transport by radiation and gas flow in the pores require a further extension of the model. For this purpose we spatially decomposed the system into simplices using a Delaunay tessellation of the particle centers. Each Delaunay simplex represents a discrete element of the pore volume and constitutes a node of the network. This node is connected to the nodes of the adjacent simplices and nodes at the corners of the simplex representing the particles. Conductances and heat capacities are determined geometrically. Our method has several advantages over conventional methods. Due to the properties of the Delaunay tessellation, we are able to resolve even small, permeable gaps between particles. Also, the total numbers of nodes and edges which determines the computing time is only defined by the number of particles and does not depend on the geometry.

\section{Acknowledgment}

This research has been supported by DFG grants WO 577/6 within the priority program SPP 1386 "Nanostructured Thermoelectric Materials: Theory, Model Systems and Controlled Synthesis", and by the grants WO 577/13 and WI 981/16-1 within the priority program SPP 1959 "Manipulation of matter controlled by electric and magnetic fields".

\section{References}

[1] Z. A. Munir et al., J. Mater. Sci. 41, 763 (2006)

[2] O. Guillon et al., Adv. Eng. Mater. 16, 830 (2014)

[3] J.E. Garay, Ann. Rev. Mater. Res. 40, 445 (2010)

[4] R. Marder et al., Scr. Mater. 82, 57 (2014)

[5] L. Wang et al., J. Asian Ceram. Soc. 3, 183 (2015)

[6] D. Giuntini et al., Materials 6, 2612 (2013)

[7] E. A. Olevsky, J. Am. Ceram. Soc. 95, 2414 (2012)

[8] D. Schwesig et al., Nanotechnology 22, 135601 (2011)

[9] S. Angst et al., in AIP Conference Proceedings (AIP Publishing, 2013), Vol. 1542, p. 593

[10] D. E. Wolf et al., in Nanoparticles from the Gasphase, edited by A. Lorke et al. (Springer, Berlin, 2012), p. 161

[11] C. Goupil et al., Entropy 13, 1481 (2011)

[12] S. Angst et al., New J. Phys. 18, 043004 (2016)

[13] D. Gautam et al., J. Mater. Chem. A 3, 189 (2014)

[14] M.L. Eggersdorfer et al., Langmuir 27, 6358 (2011) 